\title{
Violência sexual contra crianças e adolescentes: políticas de prevenção e enfrentamento
}

\author{
Sexual violence against children e adolescents: prevention and coping policies
}

Violencia sexual contra ninõs y adolecentes: políticas de prevención y afrontamiento

Magna Terra Jordão ${ }^{1 *}$, Luiz Claudio Alves Braz1, Luana Frigulha Guisso', Linccon Fricks Hernandes $^{2}$, João Antônio Gonçalves ${ }^{3}$.

\begin{abstract}
RESUMO
Objetivo: Discutir o Projeto de Disseminação do Plano Nacional de Enfrentamento da Violência Sexual contra Crianças e Adolescente. Revisão bibliográfica: Realizou-se uma revisão narrativa de literatura em obras literárias e artigos científicos que apontam os problemas causados pela violência sexual contra crianças e adolescentes. A ideia gira em torno das propostas de políticas públicas de enfrentamento como alternativa, buscando reduzir os danos causados as vítimas, já que as políticas mais comuns são punitivas, e não resguardam previamente a integridade do menor. Para tal, buscou-se discutir de maneira revisional o Plano Nacional de Enfrentamento da Violência Sexual contra Crianças e Adolescente à fim de encontrar alternativas para o problema em questão. Considerações finais: Constatou-se dificuldades em nível nacional na aplicação do plano, assim como ausência de comitês de enfrentamento e prevenção a violência sexual de crianças e adolescentes em vários estados brasileiros, o que enfraquece a rede de proteção aumentando a vulnerabilidade e impunidade dos casos, assim como ausência de serviços para este público.
\end{abstract}

Palavras-chave: Violência sexual, Políticas públicas, Crianças, Adolescentes.

\begin{abstract}
Objective: To discuss the Dissemination Project of the National Plan to Combat Sexual Violence against Children and Adolescents. Bibliographic review: A narrative literature review was carried out on literary works and scientific articles that point out the problems caused by sexual violence against children and adolescents. The idea revolves around proposals for public coping policies as an alternative, seeking to reduce the damage caused to the victims, since the most common policies are punitive, and do not previously protect the integrity of the child. To this end, we sought to discuss the National Plan to Combat Sexual Violence against Children and Adolescents in a revisional manner in order to find alternatives to the problem in question. Final considerations: Difficulties were found at the national level in the implementation of the plan, as well as the absence of committees to confront and prevent sexual violence against children and adolescents in several Brazilian states, which weakens the protection network, increasing the vulnerability and impunity of cases. , as well as the lack of services for this public.
\end{abstract}

Key words: Sexual violence, Public policies, Children, Adolescents.

\section{RESUMEN}

Objetivo: Discutir el Proyecto de Difusión del Plan Nacional para Combatir la Violencia Sexual contra Niños, Niñas y Adolescentes. Revisión bibliográfica: se realizó una revisión de la literatura narrativa sobre obras literarias y artículos científicos que señalan los problemas causados por la violencia sexual contra niños y adolescentes. La idea gira en torno a propuestas de políticas públicas de afrontamiento como una alternativa, buscando reducir el daño causado a las víctimas, ya que las políticas más comunes son punitivas y no protegen previamente la integridad del niño. Con este fin, buscamos discutir el Plan Nacional para Combatir la Violencia Sexual contra Niños, Niñas y Adolescentes de forma revisada para encontrar

${ }^{1}$ Faculdade Vale do Cricaré (FVC), São Mateus - ES. *E-mail: magna.terra@hotmail.com Escola Superior de Ciências da Santa Casa de Misericórdia de Vitória (EMESCAM), Vitória - ES. ${ }^{3}$ Centro Universitário Salesiano (UniSales), Vitória - ES. 
alternativas al problema en cuestión. Consideraciones finales: se encontraron dificultades a nivel nacional en la implementación del plan, así como la ausencia de comités para enfrentar y prevenir la violencia sexual contra niños y adolescentes en varios estados brasileños, lo que debilita la red de protección, aumentando la vulnerabilidad y la impunidad de los casos, así como la falta de servicios para este público.

Palabras clave: Violencia sexual, Políticas públicas, Niños, Adolescentes.

\section{INTRODUÇÃO}

A violência caracteriza-se como um fenômeno multifacetado, polissêmico, demasiado e desafiador que se encontra vinculado a outros temas correlatos, como o uso de substâncias psicoativas, desemprego, e as desigualdades sociais. Trata-se de uma das expressões da questão de forte impacto na sociedade contemporânea em suas distintas manifestações (HERNANDES LF, 2016).

Nesse sentido, a violência sexual contra crianças e adolescentes é outro desafio no campo da educação, configurada como um fenômeno social e também precisa ser encarada como um problema de saúde pública, pois afeta a saúde física e mental das vítimas e membros da família. Esse fenômeno atravessa diferentes classes sociais, culturas, gêneros e relações étnicas (PELISOLI C, et al., 2010).

O Ministério da Saúde entende que a violência sexual contra crianças e adolescentes se refere a qualquer comportamento que os torne vulneráveis, e esses comportamentos colocam em risco seus direitos humanos para desfrutar de um desenvolvimento sexual saudável, independentemente de serem praticados por membros da família ou de serem conhecidos. A violência pode acontecer com ou sem permissão pessoal do menor. É importante enfatizar que, por ser menor de idade, ele não assume nenhuma responsabilidade por suas ações e é responsabilidade do tutor ou do estado tomar as medidas legais apropriadas para garantir a segurança do jovem, bem como as condições físicas, sociais e psicológicas (BRASIL, 2013).

A ocorrência de casos de violência sexual está amplamente relacionada a outros tipos de violência relacionados (estrutural, urbano, físico, psicológico etc.). Em relação aos problemas físicos, os órgãos genitais da vítima e outras partes do corpo podem ser danificados. Portanto, esse evento pode causar problemas emocionais, tais como: distúrbios sexuais, uso de drogas, depressão, prostituição e suicídio, eles ainda têm o risco de doenças sexualmente transmissíveis, como AIDS, sífilis, gonorreia e gravidez inesperada (RAPASO FAG e COLEHO MCR, 2015).

A escola consiste em um campo de visão complexo, com a participação de indivíduos de diferentes idades, conhecimentos, funções, experiências e objetivos diferentes, mas essas pessoas estão reunidas em um local ou espaço físico definido, na tentativa de alcançar um objetivo comum por meio da ação. A escola é um dos muitos espaços, nesse caso, com base e experiência para lidar com a situação em questão. Em outras palavras, a experiência do grupo é criada linearmente em um tempo predeterminado e dividida entre "séries ou períodos", incorporados como um conjunto de condições para propostas, planejamento, execução e avaliação e o processo dinâmico do grupo, ou seja, no grupo há interesse em não influir sobre o que você se torna (SPAZIANI RB e MAIA ACB, 2015).

Por um lado, se os aspectos físicos da violência sexual contra crianças e adolescentes são visíveis através de abrasões, cortes e infecções, por outro lado, problemas emocionais deixaram consequências mais graves e profundas, encontradas em familiares, amigos e é invisível aos olhos do educador, produzindo interno, medo, vergonha, baixa autoestima, dificuldades em estabelecer laços emocionais e 0 eco da vida sexual ativa na idade adulta (ANDRADE I e BORGES LH, 2015).

Portanto, o ambiente escolar constitui uma cena em que crianças e/ou adolescentes passam a maior parte do tempo expressando sentimentos e experiências. Dessa forma, a instituição não é apenas responsável por ensinar a ler e escrever, mas também por proteger os direitos, promovendo a aceitação e a proteção das diferenças, para não violar os direitos. Portanto, cabe aos profissionais da educação observar com sensibilidade e atenção as crianças que possam apresentar as características da violência sexual, pois 
quando os alunos estão nessa situação, o processo educacional será afetado negativamente por essa experiência (MEDEIROS SA, et al, 2019).

Neste sentido, o presente artigo busca discutir Projeto de Disseminação do Plano Nacional de Enfrentamento da Violência Sexual contra Crianças e Adolescente como marco legal. Com isso apontar ações, seus avanços e limitações. Ainda nos objetivos, a pesquisa pretende apresentar dados no intuito de contribuir com a sociedade ao problematizar formas de enfrentamento a violência sexual contra crianças e adolescentes, consoantes as políticas públicas e os direitos previstos na Constituição Federativa, potencializando as ações promovidas pelos serviços especializados neste atendimento e munindo de conhecimento a população.

\section{REVISÃO BIBLIOGRÁFICA}

Desde a antiguidade a violência sexual contra crianças e adolescentes vem sendo perpetuada, a partir século XX este fenômeno recebeu maior visibilidade das políticas públicas, mas especificamente na década de 90, quando foi incluído na agenda do poder público e de movimentos socais como à luta nacional e internacional pelos Direitos Humanos preconizados na Constituição Federal Brasileira de 1988, no Estatuto da Criança e do Adolescente (ECRIAD) regulamentado pela Lei 8069/90. Outro importante marco legal a ser destacado se deu no dia 20 de novembro de 1989 na realização da Convenção Internacional dos Direitos da Criança, adotada pela Assembleia Geral das Nações Unidas (PLATT VB, et al., 2018).

Segundo Nascimento AF e Deslandes SF (2016), no ano de 1993 uma importante iniciativa no combate à violência e exploração sexual de crianças e adolescentes se deu com o advento da Comissão Parlamentar de Inquérito (CPI), a comissão ficou conhecida como "CPI da Prostituição infantil", esta comissão provocou uma mobilização nas diversas esferas municipais, estaduais e federais com a sociedade civil o Judiciário, que foram convocados a se pronunciar frente a esta causa.

Para Libório RMC (2013), trata-se de um movimento que foi responsável por trazer à tona uma série de violações de direitos de crianças e adolescentes vítimas de abuso e exploração sexual no Brasil, tais como o "Criança e Adolescente no Interior Amazônico: Injustiça e Descaso" e a série de reportagens realizadas pelo jornalista Gilberto Dimenstein (1992) no jornal Folha de São Paulo e o livro Meninas da Noite. Com isso, a sociedade teve maior visibilidade e complexidade desse fenômeno, o qual reverberou na mobilização de instituições governamentais e não governamentais à End Child Prostitution, Child Pornography and Trafficking of Children for Sexual Purposes - ECPAT (Campanha pelo Fim da Prostituição, da Pornografia Infantil e do Tráfico de Crianças para Fins Sexuais) e não governamentais, dentre elas a Organização Internacional do Trabalho (OIT) e a Organização das Nações Unidas (ONU).

Ainda segundo, Nascimento AF e Deslandes SF (2016) em agosto de 1996 acontece o I Congresso Mundial Contra a Exploração Sexual Comercial de Crianças, realizado em Estocolmo/Suécia. Neste ínterim, as problematizações que aconteçam neste evento fomentaram o documento conhecido como a Carta de Brasília, no qual o Brasil assume perante o Comitê sobre os Direitos da Criança, o UNICEF, a OIT, a UNESCO, o PNUD, a OMS, a ONU/AIDS, o ACNUR, a OIM, o Banco Mundial/FMI, a INTERPOL, a Divisão de Justiça e Prevenção do Delito das Nações Unidas (FNUAP), a Organização Mundial do Turismo, o Alto Comissionado dos Direitos Humanos das Nações Unidas, o Centro para os Direitos Humanos das Nações Unidas, a Comissão sobre Direitos Humanos das Nações Unidas e o Relator Especial sobre a Venda de Crianças e o Grupo de Trabalho sobre as Formas Contemporâneas de Escravidão compromisso brasileiro o compromisso em desenvolver ações mais incisivas no enfrentamento da violência, abuso e exploração sexual contra crianças e adolescentes.

Neste período de acordo Nascimento AF e Deslandes SF (2016), um determinante para que a temática se entra na pauta política no governo se deu em virtude de toda comoção pública e da mídia que começou a explorar o assunto. Entretanto a escassez de dados concretos era preocupante e um empasse para os gestores públicos e pesquisadores do tema. Visando um mecanismo de proteção e registro para os casos de abuso e violência sexual contra crianças e adolescentes, correlato a uma Campanha Nacional contra o 
Turismo Sexual no ano de 1997, criou-se o disque denúncia nacional a princípio coordenado pela Ong ABRAPIA (Associação Brasileira Multiprofissional de Proteção à Infância e Adolescência), posteriormente no ano de 2003, governo federal, assume a responsabilidade da coordenação realizando a modificação do número para Disque 100.

Cumpre ressaltar que ainda nos anos 90 o setor da saúde visando também mostra iniciativas com a Ficha de Notificação Compulsória, mais precisamente em 1996 na Secretária de Saúde do Estado do Rio de Janeiro. Na primeira metade dos anos 2000, o Ministério da Saúde passou a fazer uso das fichas de notificação em todo território nacional (NASCIMENTO AF e DESLANDES SF, 2016). Sobre este aspecto Lima AF e Deslandes SF (2011) assinalam que a "notificação compulsória da violência pela saúde pública demonstra o compromisso legal e assume sua responsabilidade na proteção integral de crianças e adolescentes, sendo compreendida como um instrumento disparador de ações, permitindo adotar medidas imediatas para interferir no ciclo da violência."

\section{Projeto de disseminação do Plano Nacional de Enfrentamento da Violência Sexual contra Crianças e Adolescente como marco legal (PNEVSIJ)}

O Brasil no ano de 2008 sediou o III Congresso Mundial de enfretamento de Exploração Sexual de Crianças e Adolescentes, tendo como metas: analisar os novos cenários da exploração sexual na contemporaneidade; identificar avanços e lacunas no marco legal e na responsabilização; compartilhar experiências de implementação de políticas intersetoriais; ampliar parcerias com o setor privado; definir estratégias e metas possíveis de serem pactuadas em cooperação internacional. Neste evento, ainda se problematizou a necessidade de intervir diante das novas configurações de violência sexual, os crimes transnacionais e os delitos facilitados pelas tecnologias da informação e comunicação (TICs). Com isso, várias instancias se uniram para elaborar atualização do plano (PAIXÃO ACW e DESLANDES SF, 2010).

No ano de 2010 na cidade de Natal (RN) 160 instituições compactuaram com a escrita de um documento que ficou conhecido como a "Carta de Natal" cujo teor elaborava o "Plano Nacional de Enfrentamento da Violência Sexual Infanto-juvenil" (PNEVIJ), um mês após este acontecimento foi instituído através da Assembleia Ordinária do Conselho Nacional dos Direitos da Criança e do Adolescente (CONANDA). Tornase relevante destacar que a deliberação do plano nacional pelo CONANDA num momento de grande exaltação pois comemorava-se nesta data em torno da comemoração dos dez anos do ECA, quando aproximadamente duas mil pessoas se reuniram em Brasília para o Encontro Nacional de Entidades, estavam reunidas cerca de duas mil pessoas para o Encontro Nacional de Entidades, o possibilitou que o texto do plano fosse elaborado em um curto espaço de tempo. Neste sentido, houve a participação de várias entidades e atores sociais para além dos órgãos do Executivo, Legislativo e Judiciário das três esferas da federação (PAIXÃO ACW e DESLANDES SF, 2010).

Existem inquietações ao considerar o Plano Nacional como uma política pública. A primeira pergunta se refere à estrutura do plano: em teoria, o plano se refere à política definida anteriormente, que consiste em um conjunto de diretrizes gerais, e as diretrizes gerais se referem a uma série de leis, regulamentos e conhecimentos. No que diz respeito ao PNEVSIJ, essas diretrizes políticas de referência são os princípios básicos da Lei Federal 8.069 / 90 - Regulamento de Crianças e Jovens. Essa combinação de leis, políticas e planos teve algum impacto, incluindo o tratamento do plano como uma política orientadora. A segunda pergunta diz respeito à natureza temporária do plano nacional. Geralmente, o plano tem uma duração de existência predeterminada, definida previamente com relação à meta proposta e é executada dentro de um determinado período de tempo. A primeira versão do PNEVSIJ prevê ações a serem implementadas entre 2000 e 2003. Apenas dez anos após o prazo, nossa segunda versão foi aprovada pela CONANDA em maio de 2013. Expirada a primeira edição do PNEVIJ e aprovada a segunda edição, a edição original continua sendo uma "referência" às políticas federais, estaduais e municipais (BRASIL, 2013).

No ano de 2010 o Governo Federal iniciou a segunda versão do plano, com isso visando a promoção de ações intersetoriais, foi instituído o Plano Decenal de Direitos Humanos de Crianças e Adolescentes, reeditado pelo no contexto do CONANDA no ano de $2013 \mathrm{com}$ vigência a $2020 \mathrm{com}$ vistas a guardar sintonia com o Plano Decenal, o mesmo, o Plano Nacional configura-se como um importante dispositivo 
para análise das políticas de enfrentamento e prevenção a violência sexual contra crianças e adolescentes para sociedade brasileira. Nesse sentido, o Projeto de Monitoramento dos Planos Estaduais de Enfrentamento da Violência Sexual contra Crianças e Adolescentes: Desafios e Conquistas, tem como propósito implantar e implementar dos Planos Estaduais de Enfrentamento a Violência Sexual Contra Crianças e Adolescentes, em conjunto com o Comitê Nacional de Enfrentamento e também com a Rede Acabe com a prostituição infantil, a pornografia infantil e o tráfico de crianças para propósitos sexuais (ECPAT) Brasil (EGRY EY, et al., 2018).

Os Centros de Referência Especializada em Assistência Social (CREAS), compõem rede de serviços que integram a Política de Assistência Social, são unidades públicas que prestam atendimentos famílias e pessoas que estão em situação de risco social ou tiveram seus direitos violados. No Brasil constituem como um dos principais serviços no acolhimento a crianças e adolescentes vítimas de violência sexual (SILVA RB e CEZAR PCN, 2013).

A violência sexual contra crianças e adolescentes abrangem os conceitos de abuso sexual e exploração sexual infantil. Embora dentro do senso comum para algumas pessoas e até mesmo determinados profissionais desprovidos de conhecimento os mesmos sejam considerados iguais ambos apresentam características diferentes tanto na realização do ato, como nos termos da lei. Cumpre ressaltar, que segundo a Legislação Brasileira especificamente Lei 8.069/1990 regulamenta o Estatuto da Criança e adolescente consta no Art. $2^{\circ}$ que, para os fins desta lei, as crianças são consideradas menores de 12 anos e a adolescência entre 12 e 18 (VERAS T, 2010).

\section{Dados da violência sexual infantil no mundo}

A violência acompanha a história humana e afeta todas as grupos e classes sociais. Isso também levou a um declínio na qualidade de vida de indivíduos e comunidades e, na sociedade contemporânea, constitui um sério problema de saúde pública em todo o mundo. Entre os diferentes tipos de violência, o abuso sexual sempre foi uma preocupação, e esse fenômeno é comum na sociedade em geral. Estima-se que aproximadamente 40 milhões de crianças e adolescentes em todo o mundo sejam vítimas de abuso sexual por ano. No entanto, devido à ocorrência desses incidentes, à freqüente dependência das vítimas dos agressores e o medo do constrangimento associados ao relato de tais atos de violência, essa estimativa pode ser subestimada (SANTOS MJ, et al., 2018).

O abuso sexual e a violência contra crianças e adolescentes são fenômenos globalmente devastadores que causam sérios problemas a saúde mental durante todo o processo de desenvolvimento. $O$ abuso ocorre mesmo em lugares e pessoas cuja integridade pública, física, moral e psicológica deve ser protegida.Ao tentar entender o assunto, uma grande quantidade de dados pode ser encontrada, o que indica a complexidade do abuso sexual, e a necessidade de ter uma rede que possa apaziguar e amparar a vítima, porque ela acredita em algum tipo de "segurança" e, portanto, se culpa pelo que aconteceu. Tudo, silenciosamente procurando maneiras de se proteger e procurar proteger os laços familiares (FONTES LFC, et al., 2017).

O consenso é de que os setores de educação, saúde, assistência social, segurança pública, turismo e lazer e cultura constituem instituições legais da sociedade e são estabelecidos pelo Estado para enfrentar a violência sexual contra esse grupo. Essas necessidades são respondidas na rede pública, dependendo se os profissionais são obrigados a cumprir com os regulamentos e implementar medidas de proteção para esse grupo. No entanto, a literatura indica que os profissionais que cuidam desses casos não são capazes, principalmente quando denunciam violência sexual (VIEIRA LJES, et al., 2015). Como todas as categorias de violência contra crianças e adolescentes, a violência sexual também pode incluir as seguintes subcategorias: violência doméstica, violência intra-doméstica e violência extra-doméstica. A violência doméstica é realizada em um espaço privado na residência da vítima; o agressor não é necessariamente um membro da família, eles podem ser outras pessoas que moram na mesma casa. A violência sexual dentro da família ocorre nas mãos do agressor, que tem uma proximidade ou parentesco com a vítima e tem certo controle sobre ele a partir de um nível hierárquico (pai, mãe, padrasto e tio) e tio etc. (INOUE SRV e RISTUM M, 2008). 
O abuso sexual é um fenômeno comum que afeta todas as classes sociais, raças, religiões e culturas, sem exceção. Sua verdadeira incidência é desconhecida e acredita-se que seja um dos crimes mais relatados e do mundo. Apesar da baixa taxa de reclamações, os relatos de violência sexual ainda estão aumentando, estimando-se que 12 milhões de pessoas em todo o mundo sejam afetadas por violência sexual a cada ano. Somente nos Estados Unidos, estima-se que violações únicas ocorram a cada 6,4 minutos e um quarto das mulheres experimenta algum contato sexual inaceitável durante a infância ou a adolescência. Entre as crianças, estima-se que um terço delas às vezes tem contatos com incesto. Atualmente, cerca de 200.000 crianças americanas são abusadas sexualmente a cada ano (GASPAR RS e PEREIRA MUL, 2018).

Embora a maioria das pesquisas nesta área se concentre em apenas um tipo de vitimização, os pesquisadores dizem que outros incidentes violentos também são frequentes e acompanhados de estupro sexual, e as estatísticas oficiais mostram que essa é a razão do baixo coeficiente. A impunidade não descreve a verdadeira extensão do fenômeno. Investir em pesquisas sobre formas individuais de violência pode subestimar outras experiências de violência. Os fatores que levam à violência múltipla incluem a inserção em comunidades com sérios problemas sociais, famílias em risco e ambientes familiares em coabitação caótica (OLIVEIRA JR, et al., 2014).

A complexidade desse fenômeno geralmente é tratada de maneira enganosa tanto pelo agressor quanto pela vítima, o que prova e exige que a equipe profissional que atende esses pacientes notifique o caso, principalmente devido à relevância do problema, à prioridade de cuidar da vítima requer planejamento e implementação de diferentes populações e políticas e planos estratégicos de prevenção e intervenção. Acredita-se que a notificação de violência contra crianças e adolescentes constitua a relação entre o poder do notificador e a resistência ao agressor e tente interromper a remanufatura da violência (LUNARDI PA, et al., 2017).

\section{CONSIDERAÇÕES FINAIS}

Constatou-se dificuldades em nível nacional na aplicação do plano, assim como ausência de comitês de enfrentamento e prevenção a violência sexual de crianças e adolescentes em vários estados brasileiros, o que enfraquece a rede de proteção aumentando a vulnerabilidade e impunidade dos casos, assim como ausência de serviços para este público. Cumpre ainda ressaltar que se faz necessário maior participação da sociedade civil frente a essa problemática que afeta direta e/ou indiretamente a sociedade como um todo e que não se trata apenas de um problema de violência, mas consequência do afastamento do Estado das necessidades básicas da população, gerando marginalidade e empobrecimento.

\section{REFERÊNCIAS}

1. ANDRADE I, BORGES LH. Violência sexual contra crianças. Investigações sobre violência e sociabilidade: desafios transdisciplinares, 2015;8(1): 93-110.

2. BRASIL. Relatório de monitoramento do plano nacional de enfrentamento da violência sexual contra crianças e adolescentes, 2018. p. 91.

3. BRASIL. 2013. PLANO Nacional de Enfrentamento da Violência Sexual contra Crianças e Adolescentes. Secretaria Nacional de Direitos Humanos. Disponível em: http://www.crianca.mppr.mp.br/arquivos/File/publi/sedh/08_2013_pnevsca.pdf. Acesso em: 01 de jul. 2020.

4. GASPAR RS, PEREIRA MUL.Evolução da notificação de violência sexual no Brasil de 2009 a 2013. Cadernos de Saúde Pública, 2018; 34(11): 1-14.

5. EGRY EY, et al. Notificação da violência infantil, fluxos de atenção e processo de trabalho dos profissionais da Atenção Primária em Saúde. Ciência \& Saúde Coletiva, 2018; 23(1): 83-92.

6. FONTES LFC, et al. Violência sexual na adolescência, perfil da vítima e impactos sobre a saúde mental. Ciência \& Saúde Coletiva, 2017; 22(9): 2919-2928.

7. INOUE SRV, RISTUM M. Violência sexual: caracterização e análise de casos revelados na escola. Estudos de Psicologia (Campinas), 2008; 25(1): 11-21. 
8. LIBÓRIO RMC. Violência sexual contra crianças e adolescentes: contribuições da Psicologia no processo de prevenção. Psicologia Ensino \& Formação, 2013; 4(2): 119-139.

9. LUNARDI PA, et al. Violência contra crianças e adolescentes: características dos casos notificados em um Centro de Referência do Sul do Brasil. Enfermería Global, 2017; 46(4): 419-431.

10. MEDEIROS AS, et al. 2019. A ESCOLA como espaço de prevenção da violência sexual. Simpósio, [S.I.]. Disponível em: http://revista.ugb.edu.br/index.php/simposio/article/view/1276. Acesso em: 27 abr. 2019.

11. NASCIMENTO AF, DESLANDES SF. A construção da agenda pública brasileira de enfrentamento da violência sexual infanto-juvenil. Physis, 2016; 1(26): 1171-1191.

12. OLIVEIRA JR, et al. Violência sexual e coocorrências em crianças e adolescentes:estudo das incidências ao logo de uma década. Ciência \& Saúde Coletiva, 2014; 19(3): 759-771.

13. PAIXÃO ACW, DESLANDES SF. Análise das políticas públicas de enfrentamento da violência sexual infantojuvenil. Saúde e Sociedade, 2010; 19(1): 114-126.

14. PELISOLI C, et al. Violência sexual contra crianças e adolescentes: dados de um serviço de referência. Temas em Psicologia, 2010; 18(1): 85-97.

15. PLATT VB, et al. Violência sexual contra crianças: autores, vítimas e consequências. Ciência \& Saúde Coletiva, 2018; 23(4): 1019-1031.

16. RAPOSO FAG, COELHO MCR. Violência Doméstica contra a Crianças. In: Francine Alves Gratival Raposo; Maria Carlota de Rezende Coelho. Violência Doméstica contra a Crianças, 2015; 1(15): 65-92.

17. SANTOS MJ, et al. Caracterização da violência sexual contra crianças e adolescentes na escola - Brasil, 20102014. Epidemiologia e Serviços de Saúde, 2018; 27(2): 1-9.

18. SILVA RB, CEZAR PCN. Atuação do psicólogo no CREAS em municípios de pequeno porte. Estudos Interdisciplinares em Psicologia, 2013; 4(1): 99-109.

19. SPAZIANI RB, MAIA ACB. Educação para a sexualidade e prevenção da violência sexual na infância: concepções de professoras. Revista Psicopedagogia, 2015; 32(97).

20. VERAS T. O Sistema Nacional de Combate ao Abuso e à Exploração Sexual Infantojuvenil e o Plano Nacional: um exemplo de política pública aplicada. Cadernos EBAPE.BR, 2010; 8(3): 404-421.

21. VIEIRA LJES, et al. Capacitação para o enfrentamento da violência sexual contra crianças e adolescentes em quatro capitais brasileiras. Ciência \& Saúde Coletiva, 2015; 20(11): 3407-3416. 